\title{
MIP-based sensor platforms for the detection of histamine in the nano- and micromolar range in aqueous media
}

\author{
F. Horemans ${ }^{\mathrm{a}}$, J. Alenus ${ }^{\mathrm{a}}$, E. Bongaers ${ }^{\mathrm{a}}$, A. Weustenraed ${ }^{\mathrm{a}}$, R. Thoelen ${ }^{\mathrm{b}}$, \\ J. Duchateau ${ }^{\mathrm{a}}$, L. Lutsen ${ }^{\mathrm{c}}$, D. Vanderzande ${ }^{\mathrm{a}, \mathrm{c}}$, P. Wagner ${ }^{\mathrm{a}, \mathrm{c}}$, T.J. Cleij ${ }^{\mathrm{a}, *}$ \\ ${ }^{a}$ Hasselt University, Institute for Materials Research (IMO), Agoralaan, Building D, B-3590 Diepenbeek, Belgium \\ ${ }^{\mathrm{b}}$ XIOS University College, Agoralaan, Building H, B-3590 Diepenbeek, Belgium \\ ' IMEC, Division IMOMEC, Wetenschapspark 1, B-3590 Diepenbeek, Belgium
}

\section{A R T I C L E I N F O}

\section{Article history:}

Received 21 December 2009

Received in revised form 23 April 2010

Accepted 2 May 2010

Available online 25 May 2010

\section{Keywords:}

Biosensors

Molecular imprinting

Histamine

Impedance spectroscopy

Quartz crystal microbalance

\begin{abstract}
A B S T R A C T
The need for more advanced, accurate and lower cost sensor platforms is constantly growing. However, for certain applications the already existing sensing systems based on biological recognition elements have sometimes restrictions, which limit their use. As a result, sensors with synthetic recognition elements, such as molecular imprinted polymers (MIPs), can be interesting alternatives. Molecular imprinting leads to the formation of inert polymer particles with nanocavities, which can exhibit similar selectivity and specificity to target molecules as antibodies or enzymes. It is demonstrated that MIPs can be readily incorporated into two different sensor platforms for the detection of histamine in aqueous media. The first platform is based on electrochemical impedance spectroscopy and allows for the accurate detection of histamine in the nanomolar range. The second sensing technique is based on microgravimetry and allows for the detection of histamine in the micromolar range. Using the analogous molecule histidine, it is demonstrated that both sensor platforms are specific for the detection of histamine.
\end{abstract}

(c) 2010 Elsevier B.V. All rights reserved.

\section{Introduction}

The development of innovative biosensor concepts to detect specific molecules is of significant interest. For example, with advanced diagnostic devices it should be possible to detect biomedical relevant molecules in patient samples to monitor certain health conditions at an early stage. Many known biosensor platforms make use of enzymes or antibodies in their recognition layers [1-3]. Such biosensors based on biological recognition elements can be very sensitive and specific towards their target. Notwithstanding, some significant drawbacks can be identified. First of all, it is time consuming to obtain biological recognition elements in sufficient quantities. Furthermore, it is not always possible to obtain suitable biological recognition elements to detect all desired targets [4]. Finally, biomolecules are often unstable in changing chemical and physical environments and have a limited shelf life. These drawbacks make their use only possible in physiological fluids [5] and render them less suitable to, for example, point of care diagnostics.

An answer to these shortcomings can be molecular imprinting [6]. Molecularly imprinted polymers (MIPs) are synthetic polymers containing imprinted nanocavities, which are able to specifically

\footnotetext{
* Corresponding author. Tel.: +32 11 268310; fax: +32 11268301.

E-mail address: thomas.cleij@uhasselt.be (T.J. Cleij).
}

rebind their target. After careful optimisation, their synthesis is usually comparatively straightforward and based on conventional polymer chemistry. This is especially true for the non-covalent approach $[7,8]$. In this approach, a mixture of functional monomers and target molecules will form a pre-polymerisation complex due to the non-covalent interactions between target and functional monomer. This complex is stabilised in an appropriate porogen. Crosslink monomers are added to this mixture to create a stable matrix. After radical polymerisation, a bulk polymer is obtained containing the embedded targets. Subsequently, the target is extracted from the polymer matrix resulting in nanocavities, which act as the complementary binding sites. MIPs show similar specific binding characteristics and selectivity towards their target as antibodies and have the advantage of being robust and inert over a wide range of different environments [9]. They are stable in, for example, a wide temperature and $\mathrm{pH}$ range. Furthermore, MIPs have the potential to be regenerated, which is usually not possible with biomolecules.

MIPs can be readily used for analytical separation [10], by packing the MIPs directly into suitable columns. However, incorporation into sensing devices is more complex. Various examples can be found in the literature were MIPs are integrated in different sensor systems. The two major platforms used in the literature to detect the binding events are based on optical interrogation and electrochemical detection [11-15]. There are some drawbacks associated 


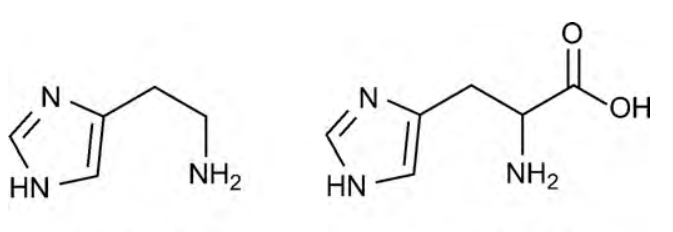

histamine

histidine

Scheme 1. Structures of histamine and histidine.

with these platforms. For example, optical and electrochemical detection often require the use of specially designed functional monomers. In addition, it is not possible to detect every type of target with these platforms, which limit their general applicability. Hence, considerable interest exits in the development of a universally applicable MIP-based sensor platform.

In our previous work, we have demonstrated a first example of a versatile biosensor platform based on MIPs, immobilized in a conjugated polymer layer [16]. Here, we present an optimised platform to which additional signal transduction capabilities are added. To this end, the MIPs are integrated into two different biosensor setups. The first setup is based on impedance spectroscopy [16-18] and makes it possible to detect targets in the nanomolar range. The other setup is based on the principle of quartz crystal microbalance (QCM) and is useful to detect targets in the micromolar range. In this way the binding events of the MIPs can be monitored over a large concentration range. Furthermore, a medically relevant target is selected, i.e. histamine (Scheme 1), which plays a crucial role in allergic reactions. For example, histamine detection is of interests for research into the Irritable Bowel Syndrome (IBS) [19] since a high concentration of histamine in the intestine can be linked to IBS.

In this article, a well-functioning biosensor is constructed for the sensitive and specific detection of histamine over a wide concentration range in vitro in an aqueous solution. In contrast, a comparable molecule, i.e. histidine (Scheme 1), does not give a significant response. Since the detection of histamine is important food science and biomedical testing, the developed sensor platforms may find use in a variety of applications. For example, typical histamine concentrations in human plasma range from approximately 1-15 nM [20]. The actual histamine concentration can be indicative of certain diseases, e.g. the histamine concentration in group of women with breast cancer was $8.23 \pm 3.4 \mathrm{nM}$, whereas the healthy control group only has a histamine concentration of $5.92 \pm 3.1 \mathrm{nM}$ in the plasma [21]. Hence, concentration detection in the nanomolar range is of significant importance. Another possible application for a histamine biosensor is the verification of the freshness of food samples. Spoiled foods contain higher histamine levels due to the degradations of proteins [22]. In this type of applications, relevant histamine concentrations are much higher. For example, fish poisoning can occur if spoiled fish contains more than $50 \mathrm{mg}$ of histamine per $100 \mathrm{~g}$ of fish [23].

There already exist several complicated and expensive techniques to detect histamine. The most common methods make use of high-pressure liquid chromatography (HPLC), gas chromatography (GC) or Enzyme-Linked Immuno Sorbent Assay (ELISA) [24,25]. Whereas these techniques need extensive sample preparation procedures and are laborious to execute, the biosensor system in this article is not only capable of detecting histamine with high sensitivity and selectivity, but also does not require complex sample preparation. It should be noted that only limited literature exists on MIPs towards histamine as the target molecule [26,27]. Only one MIP-based histamine sensor is reported in the literature, in which the MIP has been electropolymerised [28]. In the current work, a new bulk polymerized MIP has been developed and optimised for use in biosensors, which can bind histamine with a high affinity and selectivity. As a result of the use of a bulk polymerized MIP, the developed sensor methodology can be extended toward a wide range of target molecules.

\section{Materials and methods}

\subsection{Reagents}

Ethylene glycol dimethacrylate (EGDM), methacrylic acid (MAA) and dimethylsulfoxide (DMSO) were purchased from Acros. Prior to polymerisation, the stabilizers in the MAA and EGDM were removed by filtration over alumina. Azobisisobutyronitrile (AIBN) was purchased from Fluka. The target molecule histamine was obtained from Aldrich. The analogous molecule, histidine was obtained from Fluka and was used to test the specificity of the MIPs. All solvents were of analytical grade and purchased from Acros and used without further purification. The PPV derivative, $\mathrm{OC}_{1} \mathrm{C}_{10}$-PPV, which was used as the immobilization layer for the impedance based biosensor was synthesized via the sulfinyl precursor route [29]. All chemical and physical properties of this conjugated polymer were in agreement with previously reported data. The molecular weight $M_{\mathrm{w}}$ of the utilised polymer was $3.4 \times 10^{5}$ with a polydispersity of 3.9. The maximum wavelength of the $\pi-\pi^{*}$ transition was positioned at $505 \mathrm{~nm}$ in $\mathrm{CHCl}_{3}$ solution, indicating that a very low defect level was achieved. Polyvinyl Chloride (PVC), which was used as the immobilisation layer for the MIP and NIP in the QCM measurements, was obtained from Sigma-Aldrich (batch $\# 12706 \mathrm{ch} ; M_{\mathrm{w}}=8.0 \times 10^{4}$ with a polydispersity of 1.7 ). Water used for the dilution range of histamine and histidine for the QCM measurements was demineralised with a Sartorius Arium $611(18.2 \mathrm{M} \Omega \mathrm{cm})$ and degassed using a MD4 diaphragm vacuum pump from Vacuubrand. A phosphate buffered saline(PBS) solution was used for the impedance spectroscopy measurements instead of water.

\subsection{Equipment}

Optical absorption measurements for batch rebinding experiments were performed with a Varian Cary 500 UV-vis-NIR spectrophotometer. The morphology of the sensor surface was investigated using optical microscopy with a Zeiss Axiovert 40 MAT and using scanning electron microscopy (SEM) with a FEI Quanta 200 FEG. Microscope images were processed using the image analysis program Image $1.37 \mathrm{v}$ from the National Institute of Health, USA. The quartz crystal microbalance (QCM) measurements were performed using a PLO-10 phase lock oscillator from Maxtek Inc. The crystals were standard AT-cut, $5 \mathrm{MHz}$, Ti/Au polished with an active oscillation region of $34.19 \mathrm{~mm}^{2}$. The pump used during the QCM experiments was a NE-500 syringe pump from Prosense B.V. Impedance spectroscopy was performed using an Iviumstat electrochemical analyzer from Ivium Technologies B.V.

\subsection{MIP synthesis}

The MIP synthesis was optimised to obtain MIPs with a high affinity and selectivity for histamine detection in biomimetic sensors. The six most suitable MIPs are presented in Table 1 . They were made by combining MAA, EGDM, AIBN, DMSO and the target histamine in varying ratios. The final MIP(MIP3), which was utilized in all biosensor experiments described hereafter, was made according to the following procedure. A mixture of MAA (17.8 mmol), EGDM $(36 \mathrm{mmol})$ and AIBN $(0.66 \mathrm{mmol})$ was dissolved in $7 \mathrm{~mL}$ DMSO together with the template molecule histamine $(8.99 \mathrm{mmol})$. This mixture was degassed for 5 min with $\mathrm{N}_{2}$ to remove oxygen. For polymerisation the solution was placed in a sealed vial and kept in a thermostatic bath at $65^{\circ} \mathrm{C}$ for $12 \mathrm{~h}$. 
Table 1

Relative composition of selected MIPs with the corresponding amount of binding sites.

\begin{tabular}{lllll}
\hline MIP & Histamine & MAA & EGDM & $N_{\text {tot }}(\mu \mathrm{mol} / \mathrm{g})^{\mathrm{a}}$ \\
\hline MIP1 & 1 & 0.5 & 1 & 219 \\
MIP2 & 1 & 1 & 2 & 270 \\
MIP3 & 1 & 2 & 4 & 524 \\
MIP4 & 1 & 3 & 6 & 84 \\
MIP5 & 1 & 1 & 4 & 84 \\
MIP6 & 1 & 2 & 8 & 98 \\
\hline
\end{tabular}

a The total number of binding sites within the range from 1 to $100 \mathrm{mM}^{-1}$, as obtained after fitting with Freundlich isotherms.

After polymerisation the bulk polymer was grounded with a mechanical mortar for $24 \mathrm{~h}$ and sieved through a $25-\mu \mathrm{m}$ sieve. Only particles with a size smaller than $25 \mu \mathrm{m}$ were used. Subsequently, the histamine was removed from the MIP powders by consecutive Soxhlet extractions, starting with methanol $(48 \mathrm{~h})$, followed by a mixture of acetic acid/acetonitrile (1/1) (48 h) and finally again methanol $(12 \mathrm{~h})$. The extracted MIP powders were dried in vacuum for $12 \mathrm{~h}$. A non-imprinted polymer (NIP) was synthesized in the same manner, but without the presence of the target molecule.

\subsection{Sensing systems}

QCM is a technique, which makes use of the piezoelectric properties of quartz crystals. When weight is deposited onto the crystal surface, its oscillation frequency will change in accordance to the weight and it will stabilize at a new resonance frequency. The relationship between this frequency shift and the deposited weight is described by the Sauerbrey equation [30,31]. From this equation, the deposited mass can directly be calculated. For the sensor measurements, the QCM crystals were spincoated with a $0.7 \mathrm{wt}$.\% PVC solution in tetrahydrofuran (THF). This layer serves as an immobilisation layer for either the MIP or the NIP particles. These particles were applied to the polymer layer with the use of a polydimethylsiloxane (PDMS) stamp. Afterwards the crystals were placed on a hot plate with a temperature of $120^{\circ} \mathrm{C}$ for $10 \mathrm{~min}$. This temperature is above the glass transition temperature of PVC and will cause the MIP and NIP particles to embed partially into the PVC layer. After cooling to room temperature, the crystals were rinsed with isopropanol to remove excessive MIP and NIP particles and dried under nitrogen gas.

For the actual measurements, the crystals were placed in the crystal holder, which is on one side connected to the syringe pump and on the other side to an addition reservoir. Each measurement started with a base volume of $10 \mathrm{~mL}$ of water in the addition reservoir. Subsequently, this water was pumped through the crystal holder with a flow rate of $1 \mathrm{~mL} / \mathrm{min}$. When the signal stabilized, the flow was stopped and the desired amount of histamine or histidine was added to the addition reservoir. This mixture was stirred for $20 \mathrm{~s}$ with a magnetic stirrer. After $20 \mathrm{~s}$, the stirrer was stopped and the pumping was resumed at the same flow rate. Upon binding to the MIP or NIP the frequency dropped in accordance with the bound mass. After each measurement, the addition reservoir and the crystals were adequately rinsed to remove any remaining target molecules. Increasing concentrations of histamine and histidine were measured with both the MIP and the NIP covered crystals in the concentration range $1-100 \mu \mathrm{M}$ with $10 \mu \mathrm{M}$ increments.

The impedimetric sensor was build using 4 aluminium coplanar contacts (giving a total of 8 electrodes) with a thickness of $70 \mathrm{~nm}$ and an interspace of $0.6 \mathrm{~mm}$. Thin films of the conjugated polymer $\mathrm{OC}_{1} \mathrm{C}_{10}$-PPV were spincoated onto these electrodes. The obtained layers were uniform and the thickness was verified with Dektak measurements. The spincoating procedure was optimized to obtain polymer layers with a thickness of $100 \pm 5 \mathrm{~nm}$. In this way, one sample contains four sensor spots, which can be individually functionalized. The microparticles of the MIP or NIP were immobilized in the same manner as described for the QCM (vide supra). To this end, the samples were heated to a temperature of $120^{\circ} \mathrm{C}$ for $10 \mathrm{~min}$, which is above the glass transition temperature of $\mathrm{OC}_{1} \mathrm{C}_{10}$-PPV. Samples were placed in a printed circuit board (PCB) and contacts were wirebonded.

Measurements were performed in a controlled temperature environment at $37^{\circ} \mathrm{C}$ using an addition setup, which consists of a $500 \mu \mathrm{L}$ reservoir placed upon the PCB. For each sensing spot, spectra were measured sequentially at a low-frequency range from $1 \mathrm{~Hz}$ to $1 \mathrm{kHz}$ with zero bios voltage and an oscillating voltage of $50 \mathrm{mV}$. The concentration of histamine was increased in a $0-12 \mathrm{nM}$ range. A histamine concentration range of 9-31 nM was prepared in 12 equal steps. Constant volumes of $25 \mu \mathrm{L}$ of these concentrations were sequentially added to the PBS, resulting in the desired $1 \mathrm{nM}$ increments. The histidine concentrations were increased by repeated addition of $50 \mathrm{~mL}$ of a $29 \mathrm{nM}$ solution, resulting in unequal steps of 5.8, 9.7 and $12.4 \mathrm{nM}$. Time-resolved analysis of the impedance spectra occurred also at a low frequency, i.e. $213 \mathrm{~Hz}$. At low frequencies, the sensor is more sensitive for surface interactions between the MIP binding sites, the target molecules and the electrolyte [16]. It can be assumed that upon binding, electrical changes occur at that location.

\section{Results and discussion}

\subsection{Batch rebinding experiments}

The MIP synthesis has been optimised by varying the amount of MAA and EGDM. A number of MIPs and the corresponding NIPs have been synthesized with different ratios of MAA and EGDM. To characterize the binding characteristics for all prepared MIPs and NIPs, batch rebinding experiments have been performed. To this end, the MIP or NIP powder $(20 \mathrm{mg}$ ) has been added to $5 \mathrm{~mL}$ of various concentrations of histamine in aqueous solutions. The resulting suspensions have been shaken during $4 \mathrm{~h}$ on a rocking table at room temperature. After filtration the free concentration $\left(C_{\mathrm{f}}\right)$ of histamine has been measured using UV-vis spectroscopy. Subsequently, the amount of histamine bound per gram MIP has been calculated $\left(S_{\mathrm{b}}\right)$ and binding isotherms and corresponding affinity distributions for the imprinted and nonimprinted polymers towards histamine have been made. These affinity distributions are based on the Freundlich model, which is known to give a good description of the binding characteristics in MIPs as a result of the presence of a heterogeneous distribution of binding sites [16,32-34]. Using the Freundlich model, the total number of binding sites, $N_{\text {tot }}$, within the range of affinity constants $K_{\mathrm{i}}=1-100 \mathrm{mM}^{-1}$ can be calculated (Table 1) [33]. This number is a measure for the relative capacity of the individual MIPs.

From these batch rebinding experiments it becomes evident that MIP3 has the best performance for integration into biosensors. Therefore, MIP3 and its corresponding NIP counterpart have been selected for the biosensor experiments. The binding isotherms and affinity distributions of MIP3 are shown in Fig. 1. In the remainder of this article they will be referred to as MIP and NIP. The results confirm the higher binding affinity of histamine for MIP compared to the corresponding NIP. For the region between 1 and $100 \mathrm{mM}^{-1}$ the amount of binding sites for histamine is $524 \mu \mathrm{mol} / \mathrm{g}$ for MIP and $137 \mu \mathrm{mol} / \mathrm{g}$ for the corresponding NIP. This indicates that in the used integration range, the MIP has about four times more binding sites for histamine as the corresponding NIP.

One can also estimate an overall binding constant for the MIP and NIP by assuming that every binding site present has the same affinity for histamine by using a Langmuir isotherm. This assump- 

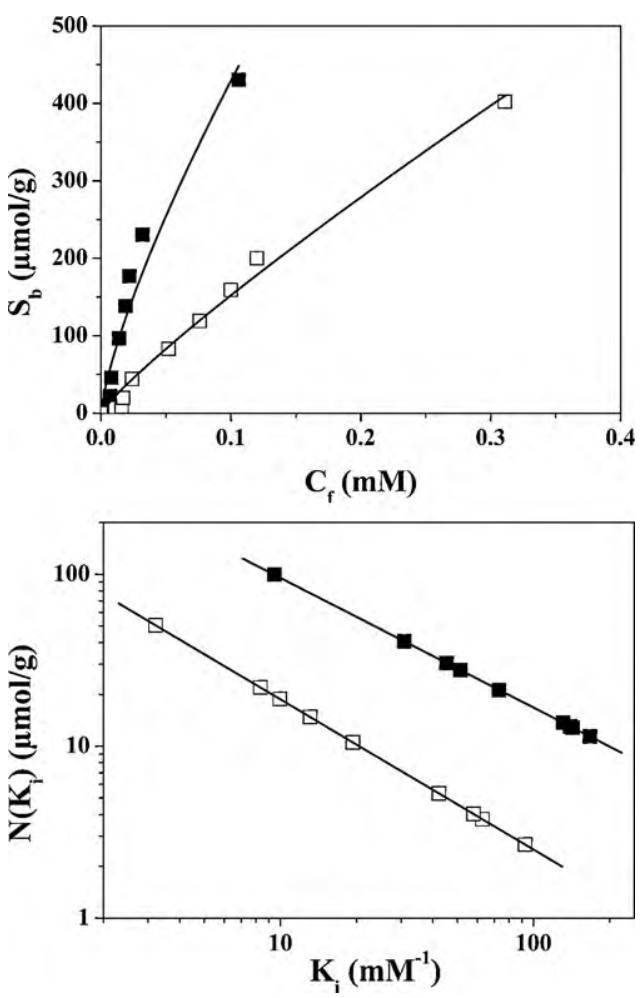

Fig. 1. Binding isotherms for MIP (solid squares) and corresponding NIP (open squares) exposed to histamine (top) and resultant affinity distributions after fitting with Freundlich isotherms (bottom).

tion is obviously incorrect, but can still provide a rough comparison of the MIP and NIP systems. In this way, binding constants for the MIP and NIP are found of 6.4 and $1.2 \mathrm{mM}^{-1}$, respectively. This indicates that, as expected, the MIP binds histamine significantly better than the NIP. This specific chemical recognition of histamine is to a large degree the result of the presence of directional hydrogen bonds in the cavities of the MIPs.

For comparison, the same MIP and NIP have also been exposed to various concentrations of histidine (Scheme 1). Histidine has a molecular structure, which is very similar to that of histamine. Hence, it can be safely assumed that if the sensor can successfully differentiate between histamine and histidine, a satisfactory specificity is achieved and that the sensor will exhibit no response to even less similar molecules. From an analysis of the binding isotherms and affinity distributions it becomes evident that the amounts of histidine bound to the MIP and NIP are more or less equal and significantly lower as compared to the histamine binding characteristics. For the region between 1 and $100 \mathrm{mM}^{-1}$ the amount of binding sites for histidine is $20 \mu \mathrm{mol} / \mathrm{g}$ for MIP and $13 \mu \mathrm{mol} / \mathrm{g}$ for the corresponding NIP, as compared to $524 \mu \mathrm{mol} / \mathrm{g}$ for the amount of histamine binding sites on the same MIP. The low binding of histidine originates from aspecific interactions with the MIP and NIP.

\subsection{Impedance spectroscopy}

Since it is evident that the optimized MIP can bind histamine in a selective manner, the MIP and NIP have been integrated into two different sensor setups. In the first setup, the chemical recognition occurring at the MIP and NIP covered surfaces is directly translated into an electrical signal using impedance spectroscopy. For the impedance measurements, the MIP and NIP are immobilized using the conjugated polymer $\mathrm{OC}_{1} \mathrm{C}_{10}-\mathrm{PPV}$, to minimize interfering resistance at the interface due to the adhesion layer. For a good (a)

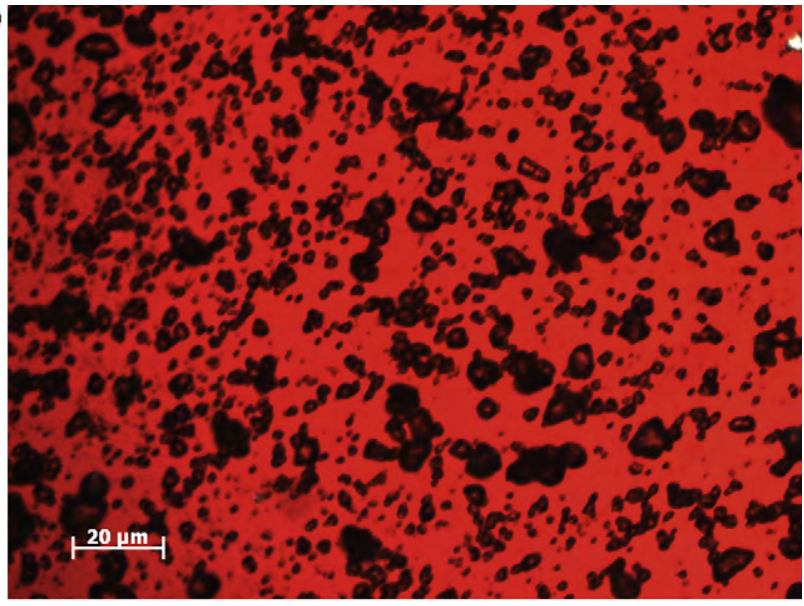

(b)

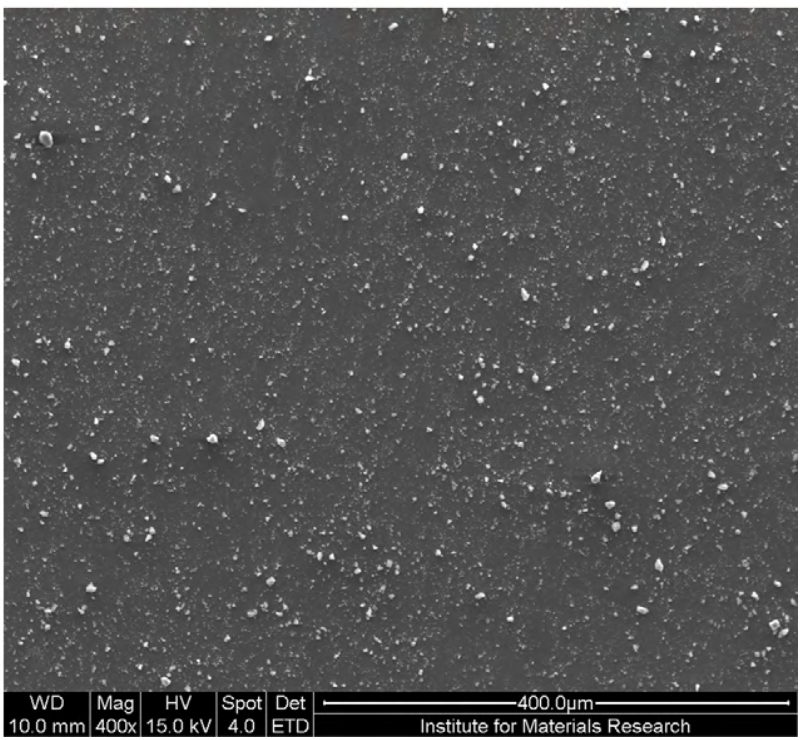

Fig. 2. (a) Image of the MIP covered surface electrode surface with the MIP particles (black) clearly visible against the conjugated polymer background (red); (b) SEM image of a small section of the MIP covered surface with the MIP particles (white) clearly visible against the conjugated polymer background (gray).

comparison of the MIP and the NIP sensor data, the immobilised amounts of the MIP and NIP on the sensor surface must be of the same order of magnitude. Hence, optical microscopy and SEM were utilized to study the MIP and NIP covered surfaces (Fig. 2). The analysis of the optical data indicates that the surface coverage is $38.6 \%$ for the MIP and $38.2 \%$ for the NIP, with some minor differences in the size and quantity of the particles. This indicates that indeed a comparable surface coverage is observed. An analysis of the SEM images provides less accurate data, since the contrast does no allow for a full differentiation between the particles and the conjugated polymer. As a result, a lower coverage is found of $16.8 \%$ for the MIP and $14.8 \%$ for the NIP. Although it can be assumed that the actual coverage is substantially higher, these images confirm the similarity between the MIP and the NIP covered surfaces.

For the actual measurements, the sensor cell is filled with PBS to simulate a biological environment. After a stabilization period of $15 \mathrm{~min}$, increasing concentrations of histamine have been added in a stepwise manner. Subsequently, the impedance is measured and a clear dose-response curve is obtained in the $0-12 \mathrm{nM}$ concentration range at a frequency of $213 \mathrm{~Hz}$ (Fig. 3). From Fig. 3, it is evident that the dose-response curve is not linear. This is a result of the fact that MIPs typically contain a heterogeneous distribution of different binding sites, with a concomitant large variety in binding 


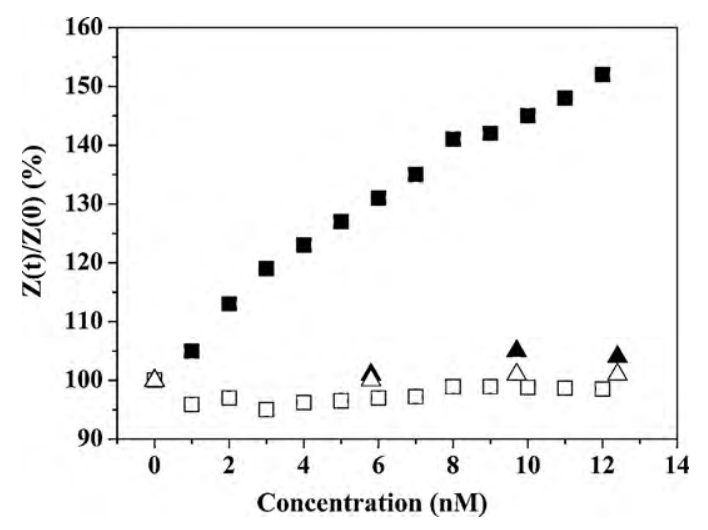

Fig. 3. Impedance spectroscopy dose-response curves for MIP and NIP exposed to increasing concentrations of histamine and histidine (MIP exposed to histamine: solid squares; MIP exposed to histidine: solid triangles; NIP exposed to histamine: open squares; NIP exposed to histidine: open triangles).

constants [33]. As a result, the dose response curve for a MIP-based sensor is expected to resemble a typical binding-isotherm, with an initial sharp somewhat linear rise, followed by gradual saturation. This is in agreement with the experimental results. It should be noted that all impedance data are normalized relative to their initial impedance value, prior to addition of the target molecule. The resulting value is referred to as the relative impedance signal. A response value is obtained $20 \mathrm{~min}$ after addition of the target molecule by averaging five data points. The stability of the measured impedance signal is excellent and as a result, the noise level is very low.

At $213 \mathrm{~Hz}$ the MIP-sensor shows a $45 \%$ response to a concentration of $10 \mathrm{nM}$ histamine. At higher concentrations, the sensor obviously will reach its saturation level. Due to the low signal to noise ration of the measurements, the limit of detection is well below $1 \mathrm{nM}$. The NIP channel, which measures non-specific binding of histamine molecules to the sensor surface, shows no sensitivity towards histamine. In addition, the specificity is tested with the analogous molecule histidine. At $213 \mathrm{~Hz}$ both the MIP and NIP channel show no response to histidine concentrations in the same nanomolar range. This confirms that the sensor is very specific for histamine and does not respond to other molecules, even if they have a somewhat similar structure. The capability of the sensor to measure in the nanomolar range is relevant for medical applications, in which deviations in the histamine concentration of a few nanomolar can be indicative of a disease (vide supra). Furthermore, it is noteworthy that our impedimetric sensor performs considerably better than a previously reported device with which a concentration limit of detection of $5 \mathrm{nM}$ was achieved under optimized flow conditions of a comparatively large sample volume [28].

\section{3. $Q C M$}

Whereas the impedimetric setup exhibit excellent performance in the nanomolar range, interest exists in a wider concentration range. To this end, a second platform for the detection of histamine has been developed based on QCM. For the QCM measurements, the quartz crystals are first coated with an adhesive polymer layer. Since no conductive properties are needed for QCM measurements, a common non-conjugated polymer, i.e. PVC, has been utilized for this layer. Subsequently, the MIPs and NIPs have been embedded into this layer by a similar stamping method as used for the impedimetric measurements, followed by a thermal treatment. After this, the resultant surface coverage has been assessed using microscopy. The surface coverage obtained from optical microscopy, is $27.1 \%$ and $28.7 \%$ for the MIP and NIP particles, respectively. Although the

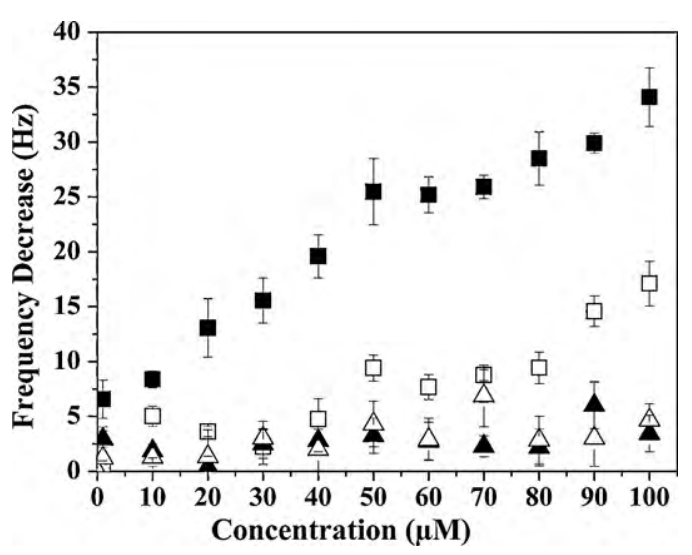

Fig. 4. QCM dose-response curves for MIP and NIP exposed to increasing concentrations of histamine and histidine (MIP exposed to histamine: solid squares; MIP exposed to histidine: solid triangles; NIP exposed to histamine: open squares; NIP exposed to histidine: open triangles).

visual distribution of the particles at the PVC surface as observed with microscopy was identical to the distribution on the impedimetric electrodes, the actual coverage values are somewhat lower than observed for the $\mathrm{OC}_{1} \mathrm{C}_{10}$-PPV immobilization (vide supra). This can be explained by the increased hardness of the PVC as compared to $\mathrm{OC}_{1} \mathrm{C}_{10}$-PPV, which apparently somewhat reduces the matrix entrapment process. It is important that the surface coverage of the MIP and NIP on each crystal is very similar, since otherwise the QCM results would be difficult to compare. After integration of the MIP and NIP covered crystals in the sensor setup, the crystals have been exposed to a series of different concentrations $(1-100 \mu \mathrm{M})$ of histamine and histidine. This resulted in typical dose-response curves (Fig. 4). Frequency shifts have been obtained after stabilization of the frequency before addition of the next concentration.

As can be seen in Fig. 4, the observed frequency shifts for the MIP exposed to histamine are significantly higher than for the NIP exposed to histamine as well as the MIP and NIP exposed histidine. For the MIP a concentration of $1 \mu \mathrm{M}$ histamine causes a frequency shift of $6.57 \mathrm{~Hz}$, which rises until the highest measured concentration of $100 \mu \mathrm{M}$ which causes a frequency shift of $34.09 \mathrm{~Hz}$. The shape of the dose-response curve is similar as observed for the impedimetric experiments. In contrast, for both the MIP and the NIP exposed to histidine the frequency remains reasonably constant (minimum $0.38 \mathrm{~Hz}$ and maximum $6.87 \mathrm{~Hz}$ ) over the entire concentration range. As expected, there are no specific binding sites for histidine and only aspecific binding is observed. This confirms the previous observations on the excellent specificity of the MIPbased sensor. Due to aspecific binding, the NIP exposed to histamine exhibits a similar behaviour, with a slightly higher frequency shift for the larger concentrations. This rise can be attributed to the significantly higher concentrations used in these experiments, as compared to the impedimetric measurements. In addition, this frequency shift associated with aspecific binding is slightly more pronounced than that observed for histidine because of the fact that the MIP synthesis has been optimised for the specific binding of histamine (vide supra). Hence, the NIP synthesized from the same optimised mixture of monomers, cross-linkers and porogen (but without the template being present), can be expected to have also a somewhat higher amount of aspecific binding sites for histamine.

For a true comparison, the corresponding mass change has been calculated for every frequency shift. It is found that the binding of $6.11 \mathrm{ng}$ of target molecules corresponds to frequency decrease of $1 \mathrm{~Hz}$. The thus obtained mass change of the sensor has been compared to the total mass that passed the sensor for that given concentration, giving an estimate of the percentage of molecules that bind to either the MIP or NIP. From these calculations it is 


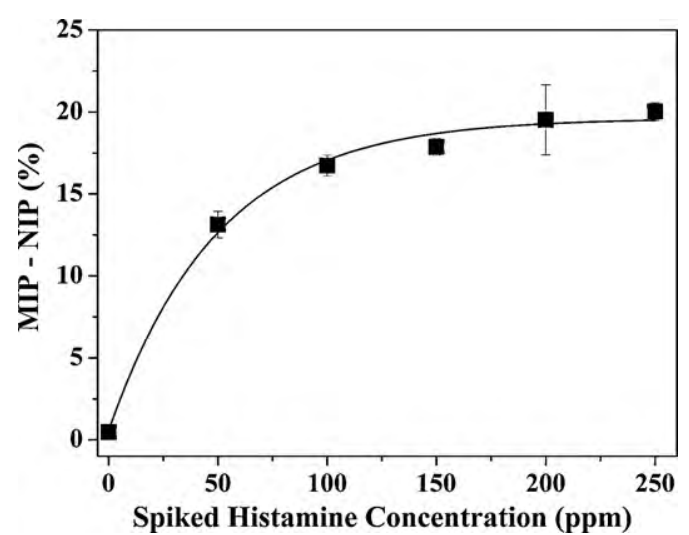

Fig. 5. Response of the MIP-based sensor to various concentrations of histamine in spiked samples of canned tuna liquid.

apparent that histamine binds about 14 times better to the MIPbased sensor surface than histidine does. Histamine binds also almost five times better to the MIP-based sensor surface as compared to the NIP. This is in agreement with the batch rebinding experiments, which demonstrated that the MIP has about four times as many binding sites for histamine as compared to the NIP. The concentration range for the QCM based sensor in the micromolar range is well suited for food sample measurements.

\subsection{Sensor performance in actual samples}

As mentioned in the introduction, histamine is commonly found in food samples. In seafood products, high levels of histamine can be associated with food spoilage and can lead to sever health problems. Generally it is found that seafood containing histamine levels of $50 \mathrm{ppm}$ or more are of unacceptable quality [35]. It is therefore important to be able to differentiate between acceptable histamine concentrations and levels above $50 \mathrm{ppm}$. To demonstrate the viability of our MIP-based sensor for such measurements, impedance measurements have been performed on the packing fluids collected from canned tuna. To this end, the collected fluid has been first filtered with a series of filters, with a last filter size of $45 \mu \mathrm{m}$. The fluid has subsequently been split into two parts and one part of the fluid has been spiked with various concentrations of histamine, i.e. 50, 100, 150, 200 and $250 \mathrm{ppm}$, while the other part remained unaltered. The unaltered samples have a histamine concentration of approximately $4 \mathrm{ppm}$. Since the histamine concentration in the spiked samples is well above the suitable detection range of our sensor, all samples have been diluted 1000 times with PBS.

Upon exposing the sensor to these diluted solutions, it becomes evident that the measurements with the spiked tuna samples exhibit a considerable larger response than the unaltered sample. For example, for the tune fluid sample spiked with $250 \mathrm{ppm}$ histamine, an increase in the impedance is found of 33.0\%, while after addition, of the non-spiked sample only an increase is found of $12.8 \%$, which is probably associated with differences in the composition of the fluid in the measurement cell due to the tuna fluid addition. The NIP exhibits only a minor increase in response, which is a result of aspecific adsorption (vide supra). Hence, for a true representation of the specific binding events, the NIP signal is also in this case subtracted from the MIP signal. The results are presented in Fig. 5.

Upon spiking with $50 \mathrm{ppm}$ a large increase in the corrected sensor signal is visible. However, spiking with higher concentrations leads to a gradual saturation of the sensor. Notwithstanding, it is clearly demonstrated that the sensor performs well with an actual sample. This proof of principle, in which the sample has been diluted 1000 times, further indicates that it is possible to mea- sure histamine concentrations of $50 \mathrm{ppb}$, and possibly much lower, in actual samples using the MIP-based sensor setup without any problems.

\section{Conclusion}

MIPs with high affinity and specificity towards histamine have been developed for sensor applications. Executing batch rebinding experiments to characterize the target binding phenomena has proven to be a suitable way to screen the MIPs and the corresponding NIPs. In this manner, it is possible to predict, which MIP is most useful for integration into the biosensor systems. The optimised MIP binds significantly more histamine as compared to the corresponding NIP. The limited binding on the NIP is governed by aspecific interactions between histamine and the polymer matrix. In contrast, the binding isotherms for the MIP and corresponding NIP towards the analogous molecule histidine show even less aspecific binding. Both the optimised MIP and the corresponding NIP have been successfully integrated as synthetic recognition elements for the detection of histamine into two sensing platforms, i.e. QCM and impedimetric detection. Whereas QCM can be successfully used to detect histamine in the micromolar range, impedance based measurements make it possible to detect the target in the nanomolar range. Using the analogous molecule histidine, it is demonstrated that both sensor platforms have a high specificity for the detection of histamine. In conclusion, it has been demonstrated that using our MIP-based sensor platforms, histamine can be readily detected over a large concentration range from $1 \mathrm{nM}$ to $100 \mu \mathrm{M}$ in a sensitive and selective manner. This opens the way for many other novel MIP-based sensor applications. As a first proof of principle, the measurement of histamine in the liquid of canned tuna is demonstrated using the MIP-based sensor.

\section{Acknowledgements}

This work is supported by the School for Life Sciences of the transnational University Limburg and Hasselt University via the BOF-project "Development and Characterisation of Fluorescent Molecularly Imprinted Polymers for Nanotechnology and Applications in Advanced Electronic Devices, Chemo- and Bio-sensors" and the Fund for Scientific Research Flanders via the Scientific Research Community FWO-WOG (W0.035.04N) "Hybrid systems at nanometer scale". The authors would like to thank F.J. Troost and R.-J.M. Brummer of Maastricht University for the stimulating discussions. In addition, technical assistance by H. Penxten, J. Sogen and J. Baccus (Hasselt University) is gratefully acknowledged.

\section{References}

[1] T. Vo-Dinh, B. Cullum, Biosensors and biochips: advances in biological and medical diagnostics, Fresenius J. Anal. Chem. 366 (2000) 540-551.

[2] F. Ricci, G. Volpe, L. Micheli, G. Palleschi, A review on novel developments and applications of immunosensors in food analysis, Anal. Chim. Acta 605 (2007) 111-129.

[3] P.D. Skottrup, M. Nicolaisen, A.F. Justesen, Towards on-site pathogen detection using antibody based sensors, Biosens. Bioelectron. 24 (2008) 339-348.

[4] K. Yano, I. Karube, Molecularly imprinted polymers for biosensor applications, Trends Anal. Chem. 18 (1999) 199-204.

[5] V.B. Kandimalla, H. Ju, Molecular imprinting: a dynamic technique for diverse applications in analytical chemistry, Anal. Bioanal. Chem. 380 (2004) 587-605.

[6] C. Alexander, H.S. Andersson, L.I. Andersson, R.J. Ansell, N. Kirsch, I.A. Nicholls, J. O'Mahony, M.J. Whitcombe, Molecular imprinting science and technology: a survey of the literature for the years up to and including 2003, J. Mol. Recognit. 19 (2006) 106-180.

[7] B. Sellergren, C.J. Allender, Molecularly imprinted polymers: a bridge to advanced drug delivery, Adv. Drug Deliv. Rev. 57 (2005) 1733-1741.

[8] H. Yan, K.H. Row, Characteristic and synthetic approach of molecularly imprinted polymer, Int. J. Mol. Sci. 7 (2006) 155-178.

[9] P.K. Owens, L. Karlsson, Molecular imprinting for bio-, and pharmaceutical analysis, Trends Anal. Chem. 18 (1999) 146-154. 
[10] E. Benito-Peña, J.L. Urraca, B. Sellergren, M.C. Moreno-Bondi, Solid-phase extraction of fluoroquinolones from aqueous samples using a watercompatible stochiometrically imprinted polymer, J. Chromatogr. A 1208 (2008) 62-70.

[11] M. Avila, M. Zougagh, A. Escarpa, A. Rios, Molecularly imprinted polymers for selective piezoelectric sensing of small molecules, Trends Anal. Chem. 27 (2008) 54-65.

[12] S.M. Ng, R. Narayanaswamy, Fluorescence sensor using a molecularly imprinted polymer as a recognition receptor for the detection of aluminium ions in aqueous media, Anal. Bioanal. Chem. 386 (2006) 1235-1244

[13] L. Yang, W. Wei, J. Xia, H. Tao, P. Yang, Capacitive biosensor for glutathione detection based on electropolymerized molecularly imprinted polymer and kinetic investigation of the recognition process, Electroanalysis 17 (2005) 969-977.

[14] T.A. Sergeyeva, S.A. Piletsky, A.A. Brovko, E.A. Slinchenko, L.M. Sergeeva, T.L. Panasyuk, A.V. El'skaya, Conductimetric sensor for atrazine detection based on molecularly imprinted polymer membranes, Analyst 124 (1999) 331-334.

[15] O.Y.F. Henry, S.A. Piletsky, D.C. Cullen, Fabrication of molecularly imprinted polymer microarray on a chip by mid-infrared laser pulse initiated polymerisation, Biosens. Bioelectron. 23 (2008) 1769-1775.

[16] R. Thoelen, R. Vansweevelt, J. Duchateau, F. Horemans, J. D'Haen, L. Lutsen, D. Vanderzande, M. Ameloot, M. vandeVen, T.J. Cleij, P. Wagner, A MIP-based impedimetric sensor for the detection of low-MW molecules, Biosens. Bioelectron. 23 (2008) 913-918.

[17] P. Cooreman, R. Thoelen, J. Manca, M vandeVen, V. Vermeeren, L. Michiels, M. Ameloot, P. Wagner, Impedimetric immunosensors based on the conjugated polymer PPV, Biosens. Bioelectron. 20 (2005) 2151-2156.

[18] V.Vermeeren, N. Bijnens, S. Wenmackers, M. Daenen, K. Haenen, O.A. Williams, M. Ameloot, M. vandeVen, P. Wagner, L. Michiels, Towards a real-time, labelfree, diamond-based DNA sensor, Langmuir 23 (2007) 13193-13202.

[19] G. Barbara, V. Stanghellini, R. Giorgio, C. Cremon, G.S. Cottrell, D. Santini, G. Pasquinelli, A.M. Morselli-Labate, E.F. Grady, N.W. Bunnett, S.M. Collins, R. Corinaldesi, Activated mast cells in proximity to colonic nerves correlate with abdominal pain in irritable bowel syndrome, Gastroenterology 126 (2004) 693-702.

[20] L.G. Harsing, H. Nagashima, D. Duncalf, E.S. Vizi, P.L. Goldiner, Determination of histamine concentrations in plasma by liquid chromatography/electrochemistry, Clin. Chem. 32 (1986) 1823-1827.

[21] J. Von Mach-Szczypiński, S. Stanosz, K. Sieja, M. Stanosz, Metabolism of histamine in tissues of primary ductal breast cancer, Metabolism 58 (2009) 867-870.

[22] L. Auerswald, C. Morren, A.L. Lopata, Histamine levels in seventeen species of fresh and processed South African seafood, Food Chem. 98 (2006) 231-239.

[23] L. Lehane, J. Olley, Histamine fish poisoning revisited, Int. J. Food. Microbiol. 58 (2000) 1-37.

[24] F. Gosetti, E. Mazzucco, V. Gianotti, S. Polati, M.C. Gennaro, High performance liquid chromatography/tandem mass spectrometry determination of biogenic amines in typical Piedmont cheeses, J. Chromatogr. A 1149 (2007) 151-157.

[25] M. Claeys-Bruno, O. Vandenabeele-Trambouze, M. Sergent, M. Geffard, D. Bodet, M. Dobrijevic, A. Commeyras, R. Phan Tan Luu, Methodological approaches for histamine quantification using derivatization by chloroethylnitrosourea and elisa measurement. Part II Optimisation of the derivatization step, Chemom. Intell. Lab. Syst. 80 (2006) 186-197.

[26] C.J. Allender, C. Richardson, B. Woodhouse, C.M. Heard, K.R. Brain, Pharmaceutical applications for molecularly imprinted polymers, Int. J. Pharm. 195 (2000) 39-43.

[27] A. Tong, H. Dong, L. Li, Molecular imprinting-based fluorescent chemosensor for histamine using zinc(II)-protoporphyrin as a functional monomer, Anal. Chim. Acta 466 (2002) 31-37.

[28] A. Pietrzyk, S. Suriyanarayanan, W. Kutner, R. Chitta, F. D'Souza, Selective histamine piezoelectric chemosensor using a recognition film of the molecularly imprinted polymer of bis(bithiophene) derivatives, Anal. Chem. 81 (2009) 2633-2643.

[29] F. Louwet, D. Vanderzande, J. Gelan, A general synthetic route to high molecular weight poly(p-xylylene)-derivatives: a new route to poly(p-phenylene vinylene), Synth. Met. 69 (1995) 509-510.

[30] K. Reimhult, K. Yoshimatsu, K. Risveden, S. Chen, L. Ye, A. Krozer, Characterization of QCM sensor surfaces coated with molecularly imprinted nanoparticles, Biosens. Bioelectron. 23 (2008) 1908-1914.

[31] G. Sauerbrey, Verwendung von schwingquarzen zur wägung dünner schichten und zur mikrowägung, Z. Phys. 155 (1959) 206-222.

[32] R.J. Umpleby, M. Bode, K.D. Shimizu, Measurement of the continuous distribution of binding sites in molecularly imprinted polymers, Analyst 125 (2000) 1261-1265.

[33] D.A. Spivak, Optimization, evaluation, and characterization of molecularly imprinted polymers, Adv. Drug Deliv. Rev. 57 (2005) 1779-1794.
[34] H. Freundlich, Über die adsorption in lösungen, Z. Phys. Chem. 57 (1906) 385-470.

[35] P.L. Rogers, W.F. Staruszkiewicz, Histamine Test Kit Comparison, J. Aquat. Food Prod. Technol. 9 (2000) 5-17.

\section{Biographies}

Frederik Horemans is a PhD student at the Institute for Material Research (IMO) of Hasselt University. He is currently working on molecular imprinting for biosensor applications. He graduated from Hasselt University in 2006 with a master degree in bio-electronics and nanotechnology.

Jan Alenus is a PhD student at the Institute for Material Research (IMO) of Hasselt University. He is currently working on the development of new biosensor architectures. He graduated from Hasselt University in 2007 with a master degree in bio-electronics and nanotechnology.

Evi Bongaers obtained her PhD in science working in the field of biosensors at Hasselt University in 2009. She graduated from Eindhoven University of Technology in 2005 with a master degree in applied physics. She is currently working as an application scientist at SkyScan NV.

Ans Weustenraed is a $\mathrm{PhD}$ student at the Institute for Material Research (IMO) of Hasselt University. She is currently working on the use of molecular imprinting for separation purposes. She graduated from Hasselt University in 2008 with a master degree in bio-electronics and nanotechnology.

Ronald Thoelen obtained his $\mathrm{PhD}$ in the field of biosensors at Hasselt University in 2008. He is currently an assistant professor at XIOS Hogeschool Limburg.

Jan Duchateau obtained his $\mathrm{PhD}$ in the field of organic and polymer chemistry at Hasselt University in 2009. He is currently working as a Chemical Development Engineer at Sabic Europe (Geleen, The Netherlands).

Laurence Lutsen is group leader in "Organic and Polymer Materials" of the IMEC IMOMEC in Diepenbeek (Belgium). She obtained her Engineer Diploma in Chemistry with a specialization in polymers and organic materials from Ecole Nationale Superieure de Chimie de Clermont-Ferrand in 1991 and her PhD in Chemistry with a specialization in Organometallic Precursors of Materials from Université de Montpellier in 1994. After a first post-doc position at the University of Kent at Canterbury where she worked on the development of novel block copolymers with applications in microlithography, she started a second post-doc position at the Limburg Universitair Centrum (Belgium), where she worked on the design and synthesis of conjugated polymers for application in light emitting diodes. She has published more than 90 papers.

Dirk Vanderzande obtained his PhD in 1986 at the Catholic University Leuven (Belgium) in natural product synthesis. Since 1987 he has been appointed at Hasselt University (Belgium). He is a full professor with expertise in the field of organic and polymer synthesis, with special emphasis on the development of organic semiconductors for application in plastic solar cells. As a result of this expertise, his research group is prominently present in both national and European networks and projects concerning the development of organic materials for plastic electronics. He has published more than 170 papers on organic and polymer chemistry.

Patrick Wagner obtained his PhD in 1994 at Technical University Darmstadt (Germany) in experimental solid state physics with a focus on cuprate superconductors. From 1995 until 2001, he was postdoctoral researcher in the Laboratory of Solid State Physics and Magnetism at Catholic University Leuven (Belgium), where he studied the magneto-transport properties of mixed-valency magnetic oxides. Since 2001, he is a professor of physics at Hasselt University (Belgium) and responsible for the development of label-free readout techniques for DNA-, protein-, and smallmolecule sensors. Patrick Wagner is recipient of a WE Heraeus award, a Marie-Curie Fellowship of the European Union, a Methusalem Grant of the Flemish Government and is past president of the Belgian Physical Society.

Thomas Cleij obtained his PhD in 1999 at Utrecht University (The Netherlands) in polymeric materials science. After a stay of three years in the United States as an assistant professor at Louisiana State University, he is currently appointed as a full professor of organic and bio-polymer chemistry at Hasselt University (Belgium) in the Institute for Material Research. The expertise of the Laboratory for Organic and Bio-Polymer Chemistry focuses on the design, synthesis, characterization and application of functional polymeric materials. State of the art facilities for polymer synthesis and characterization are available. He has published over 50 papers on polymeric materials science and engineering. 